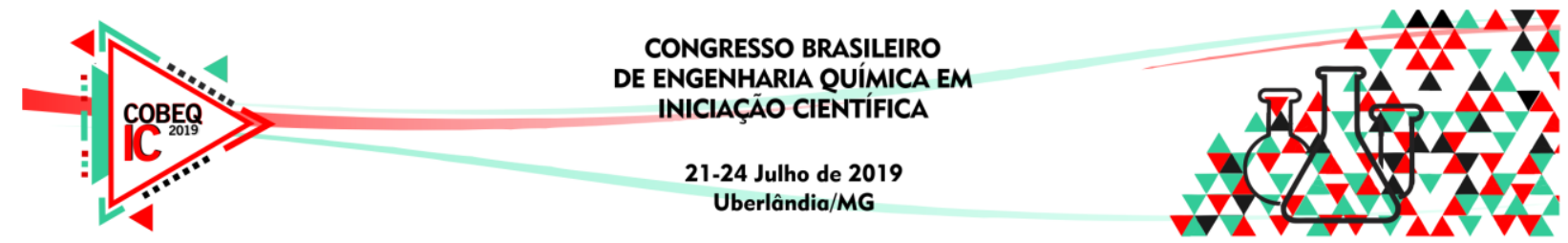

\title{
CARACTERIZAÇÃO DA CINZA DE SERRAGEM DE MADEIRA POR FLUORESCÊNCIA DE RAIOS-X E ESPECTROSCOPIA RAMAN
}

\author{
L.S. SEVERO ${ }^{1}$, D.M. PEREZ ${ }^{1}$, D.A. CAMPANELLI' ${ }^{2}$, J.B. RODRIGUES ${ }^{1}$ e L.E.G. \\ ARMAS $^{1}$ \\ ${ }^{1}$ Universidade Federal do Pampa, Programa de Pós-Graduação em Engenharia \\ ${ }^{2}$ Universidade Federal do Pampa, Faculdade de Engenharia Mecânica \\ E-mail para contato: lisiane1994@ hotmail.com
}

\begin{abstract}
RESUMO - O objetivo desse trabalho é sintetizar e caracterizar a cinza de serragem de madeira (CSM, resíduos provenientes de madeireiras), por espectroscopia de Fluorescência de raios X (FRX) e Raman confocal, com a finalidade de obter informações da qualidade e quantidade do carbono presente nas diferentes amostras, as quais posteriormente serão usadas para produzir grafeno. Para cumprir com este objetivo a serragem de madeira foi queimada em um forno mufla $(\mathrm{FM})$, em diferentes temperaturas $\left(300^{\circ} \mathrm{C}, 400^{\circ} \mathrm{C}\right.$ e $\left.500^{\circ} \mathrm{C}\right) \mathrm{e}$ tempo controlado (40,60, 120 minutos). Medidas de FRX permitiram verificar a composição química do material, a qual varia de acordo com a temperatura e tempo de carbonização. Os resultados de espectroscopia Raman mostram a presença das bandas $\mathrm{D}\left(\sim 1350 \mathrm{~cm}^{-1}\right)$ e $\mathrm{G}\left(\sim 1580 \mathrm{~cm}^{-1}\right)$, as quais são características de materiais carbonosos. Portanto, de acordo com esses resultados, concluímos que o material é adequado para a produção de grafeno. Contribuindo, dessa maneira com a redução do descarte inadequado, que podem ocasionar impactos ambientais, como a poluição de afluentes, solos, etc., visto que, apesar do material ser biodegradável sua decomposição completa leva um tempo.
\end{abstract}

\section{INTRODUÇÃO}

As pesquisas científicas na área de nano materiais, estão em constante crescimento. Pois é possível desenvolver materiais com alta durabilidade, alta resistência e de baixo impacto ambiental, assim como reduzir os custos utilizando resíduos naturais. Os estudos mostram que a utilização da serragem, provenientes da madeira se apresentam como materiais alternativos para a obtenção de nanoestruturas carbonosas.

De acordo com Bernd et. al (2017), a serragem de madeira é composta por celulose e lignina, as quais possuem grande concentração de carbono, podendo assim ser utilizada para produzir materiais carbonosos, na forma de micro e nanoestruturas.

Geim (2009) e Novoselov (2012) produziram pela primeira vez em 2004 o Grafeno, o qual é uma folha plana composta apenas por átomos de carbono ligados entre si em um 


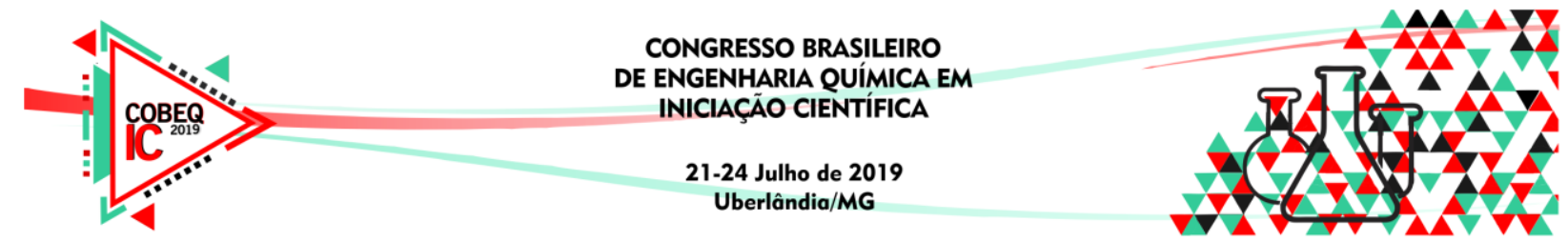

arranjo bidimensional (2D), com geometria hexagonal, formando uma folha nanométrica de átomos bem organizados e ligados pela hibridização $\mathrm{sp}^{2}$. A ligação química e a configuração eletrônica são as características cruciais para as diversas propriedades do grafeno. Após a descoberta, vários estudos estão sendo feitos usando biomassas como fonte precursora de carbono para a produção de grafeno em grande escala e baixo custo.

Portanto, neste trabalho ensaios experimentais foram realizados para analisar se a serragem de madeira (SM) é apropriada para a produção de nanoestruturas de carbono (grafeno). A carbonização do material foi realizada a diferentes temperaturas, com tempo controlado, usando um forno Mufla (FM). Após o processo de queima, as respectivas amostras foram caracterizadas por Fluorescência de Raios-X (FRX) e Espectroscopia Raman. Estes resultados mostram que é possível usar a cinza de serragem de madeira (CSM) como fonte precursora de grafeno.

\section{MATERIAIS E MÉTODOS}

\subsection{Processo de Limpeza}

Para a realização da carbonização do material foram necessários vários processos. Primeiramente foi feita a coleta do material, serragem de madeira, fornecida pela madeireira Tiarajú, localizada na cidade de Alegrete/RS. Após a coleta, como mostra a figura 1, o material passou por um processo de limpeza para retirar as impurezas. Em seguida, a serragem foi peneirada com passagem entre $2,0 \mu \mathrm{m}$, e retida na peneira $600 \mu \mathrm{m}$, sendo que a peneiração foi feita para separar partículas maiores, e foi utilizado o material que ficou retido na peneira de $600 \mu \mathrm{m}$. Após passou por uma lavagem com água destilada (2X) para eliminar a sujeira restante, como por exemplo, poeira, materiais orgânicos etc. Ao final do processo, o material foi depositado em uma estufa para secar a uma temperatura de $105^{\circ} \mathrm{C}$ por 24 horas, após a secagem o material foi armazenado em local limpo e seco.

Figura 1- Organograma da etapa 1

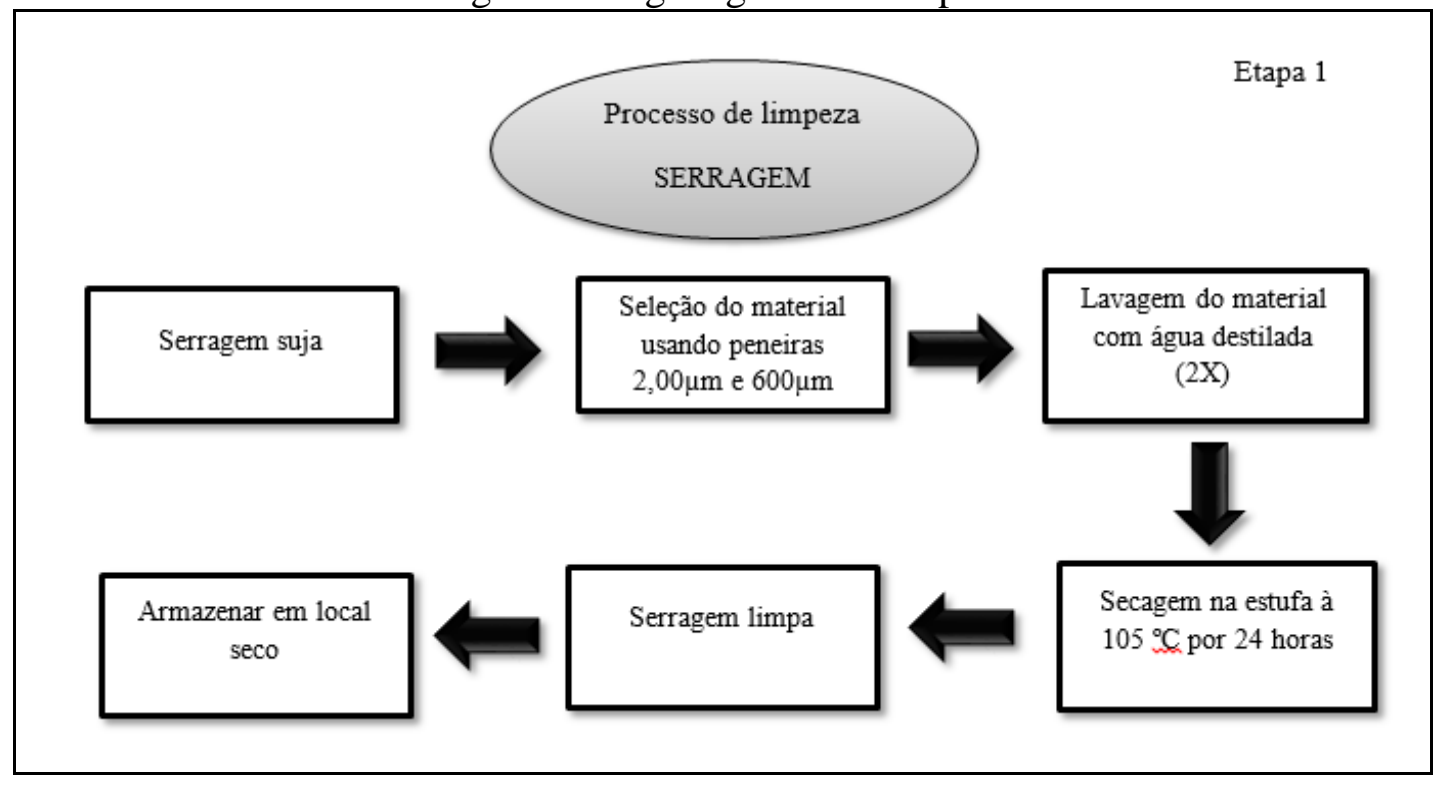




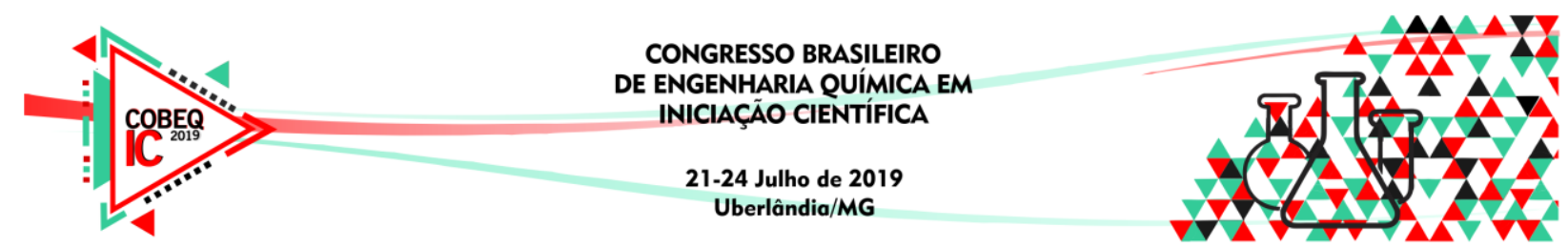

\subsection{Processo de queima}

Para o processo de queima da serragem de madeira foram usados cadinhos de porcelana. Para cada ensaio de queima usou-se cinco cadinhos, cada um com capacidade de $10 \mathrm{~g}$ aproximadamente, totalizando $50 \mathrm{~g}$ de serragem, pois por cada $10 \mathrm{~g}$ do material queimado obtém-se em torno de 2 gramas de cinzas.

A figura 2 mostra o procedimento usado para a obtenção da cinza, os cadinhos utilizados foram pesados em uma balança analítica, adicionando $10 \mathrm{~g}$ de serragem e levado ao FM para a carbonização completa. Em seguida, as amostras foram queimadas em temperaturas de $300{ }^{\circ} \mathrm{C}, 400{ }^{\circ} \mathrm{C}$ e $500{ }^{\circ} \mathrm{C}$, no tempo de 40,60 e 120 minutos para cada temperatura, com a finalidade de comprovar a presença de carbono nas três temperaturas. Posteriormente, o material foi retirado e colocado em um dessecador para resfriamento a temperatura ambiente por 30 minutos. Finalmente, a cinza passou por um processo de moagem manual usando um almofariz e um pistilo formando um pó, o qual foi caracterizado por FRX e Espectroscopia Raman.

Figura 2 - Organograma da etapa

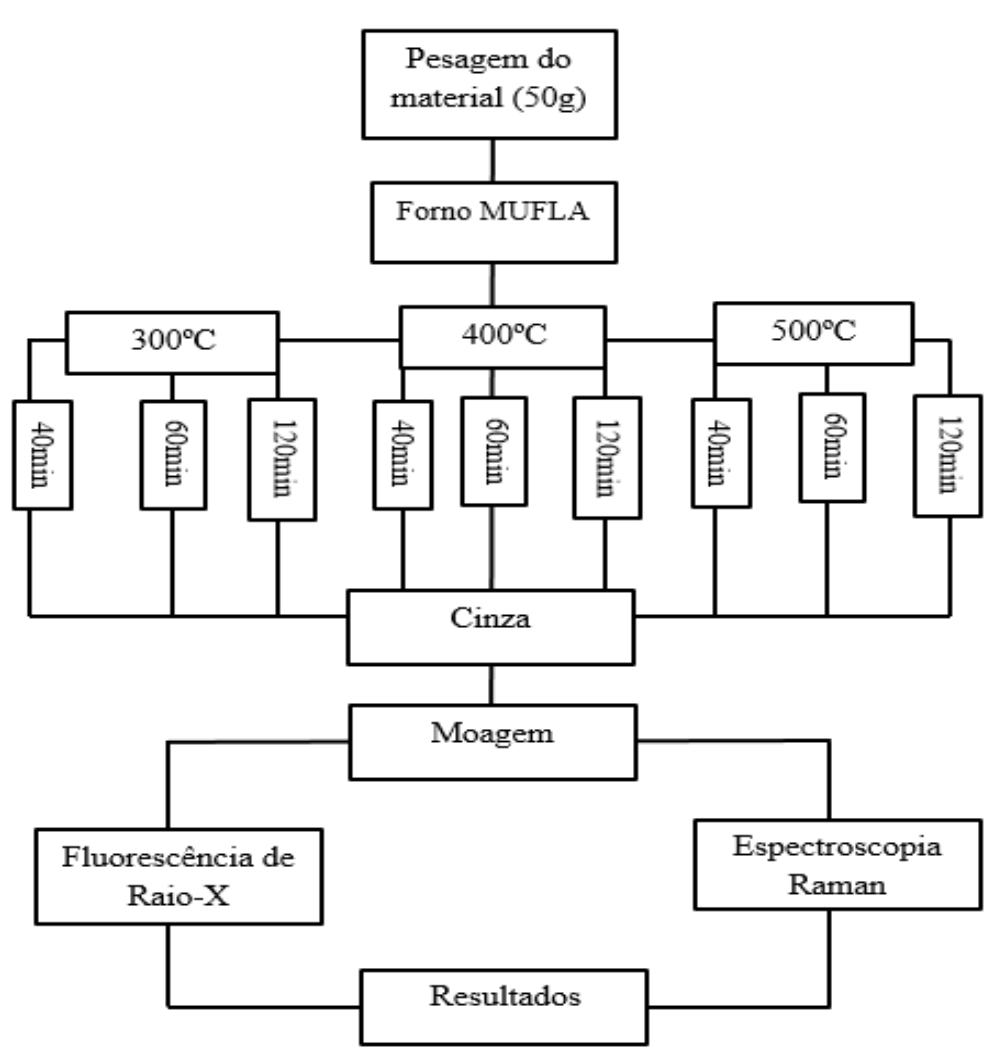




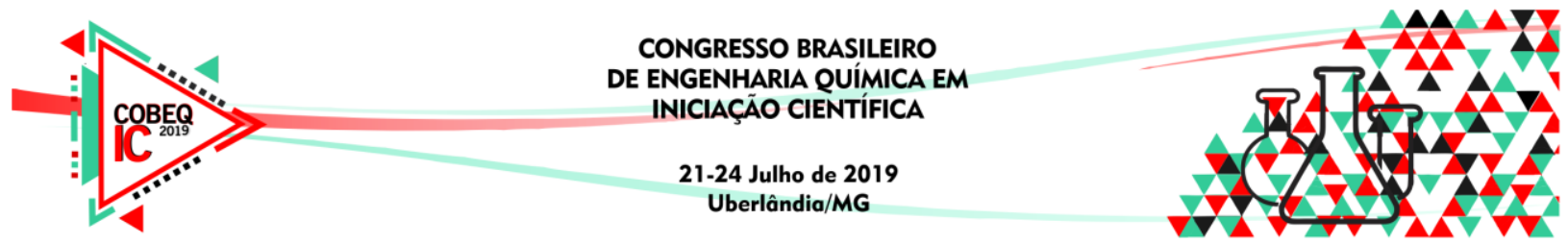

\section{RESULTADOS E DISCUSSÕES}

\subsection{Fluorescência de Raios-X}

Todas as amostras foram caracterizadas utilizando um espectrofotômetro FRX, localizado na Universidade Federal do Pampa, campus Caçapava do Sul, da marca BRUKER, modelo Turbo SD, propriedade do laboratório de Lavra, Planejamento e Tratamento de Minérios - LATRAM. É uma técnica não destrutiva que estabelece a análise quantitativa da composição química do material, e devido a limitações do equipamento, os elementos com número atômico menor ou igual $12(\mathrm{Z}=12)$ não foram detectados, como por exemplo o Carbono, Hidrogênio e Oxigênio.

Das composições químicas detectadas no material, as que apresentaram maiores porcentagens foram óxido de Silício (SiO2), óxido de Alumínio (A12O3), óxido de Cálcio $(\mathrm{CaO})$ e óxido de Magnésio $(\mathrm{MgO})$. Deve-se ressaltar que três dos compostos encontrados foram descartados, como o enxofre (S), devido aos ruídos de interferências externas durante o processo de caracterização, também elementos como o Ródio (Rh) e Cádmio (Cd) foram desconsiderados devido a erros de leitura do equipamento.

Os ensaios realizados em diferentes temperaturas mostraram que a medida que a temperatura aumenta, assim como o tempo de queima, à porcentagem dos compostos químicos variam entre um ensaio e outro, tal como mostrado na tabela 1. Nesta tabela, podese ver que o óxido de cálcio sofre uma variação significativa conforme o aumento da temperatura, de forma semelhante para os outros compostos químicos.

Tabela 1- Composição química detectada pelo FRX, em diferentes temperaturas e tempos

\begin{tabular}{|c|c|c|c|c|c|c|c|c|c|c|}
\hline \multirow{2}{*}{} & \multicolumn{9}{c|}{ Ensaios } \\
\cline { 3 - 13 } & \multicolumn{3}{|c|}{$300^{\circ} \mathrm{C}$} & \multicolumn{3}{c|}{$400^{\circ} \mathrm{C}$} & \multicolumn{3}{c|}{$500^{\circ} \mathrm{C}$} \\
\cline { 3 - 12 } & $\begin{array}{c}40 \\
\text { min }\end{array}$ & $\begin{array}{c}60 \\
\text { min }\end{array}$ & $\begin{array}{c}120 \\
\text { min }\end{array}$ & $\begin{array}{c}40 \\
\text { min }\end{array}$ & $\begin{array}{c}60 \\
\text { min }\end{array}$ & $\begin{array}{c}120 \\
\text { min }\end{array}$ & $\begin{array}{c}40 \\
\text { min }\end{array}$ & $\begin{array}{c}60 \\
\text { min }\end{array}$ & $\begin{array}{c}120 \\
\text { min }\end{array}$ \\
\hline Óxido de Magnésio & $\mathrm{MgO}$ & 2,83 & 2,48 & 1,79 & 2,38 & 1,4 & 0,81 & 2,19 & 1,53 & 0,38 \\
\hline Óxido de Alumínio & $\mathrm{Al} 2 \mathrm{O} 3$ & 3,6 & 3,74 & 3,53 & 3,45 & 3,4 & 3,45 & 3,46 & 3,45 & 3,44 \\
\hline Óxido de Silício & $\mathrm{SiO} 2$ & 0,61 & 0,59 & 0,49 & 0,53 & 2,9 & 1,23 & 0,55 & 1,30 & 0,57 \\
\hline Óxido de Potássio & $\mathrm{K} 2 \mathrm{O}$ & 0,47 & 0,4 & 0,56 & 0,48 & 0,53 & 1,13 & 0,55 & 1,32 & 0,57 \\
\hline Óxido de Cálcio & $\mathrm{CaO}$ & 2,8 & 2,98 & 3,92 & 3,56 & 4,24 & 10,15 & 3,7 & 9,77 & 4,57 \\
\hline Óxido de Ferro & $\mathrm{Fe} 2 \mathrm{O} 3$ & 0,27 & 0,18 & 0,72 & 0,35 & 0,4 & 0,94 & 0,47 & 0,94 & 0,48 \\
\hline Óxido Manganês & $\mathrm{MnO}$ & 0,06 & 0,07 & 0,09 & 0,07 & 0,08 & 0,14 & 0,1 & 0,17 & 0,08 \\
\hline
\end{tabular}

\subsection{Espectroscopia Raman}

Análises da CSM pela Espectroscopia Raman da marca HORIBA, modelo XploRA PLUS, da Universidade Federal do Pampa, campus Alegrete, identificou a presença da banda $\mathrm{D}\left(\sim 1357 \mathrm{~cm}^{-1}\right)$ e banda $\mathrm{G}\left(\sim 1590 \mathrm{~cm}^{-1}\right)$ nas diferentes temperaturas $\left(300,400\right.$ e $\left.500^{\circ} \mathrm{C}\right)$ característicos de materiais carbonosos. Através desses resultados é possível inferir a aplicação do material para a síntese de grafeno. Os espectros analisados na figura 3 mostram a 


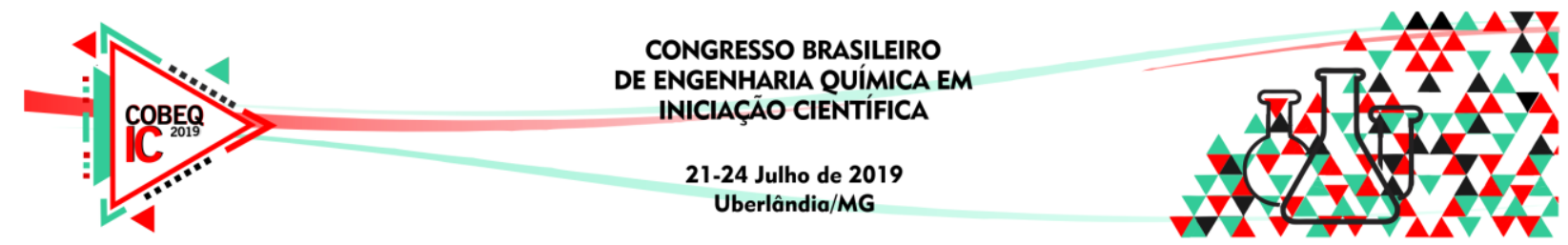

presença das bandas ( $\mathrm{D}$ e $\mathrm{G}$ ), a qual refere-se a presença de carbono nas amostras caracterizadas.

A presença de carbono nas amostras está relacionada com a composição química da serragem de madeira, como a celulose e lignina principais componentes da madeira a qual possuem em sua estrutura molecular uma elevada concentração de carbono. A decomposição completa da celulose acontece entre 300 e $500^{\circ} \mathrm{C}$, através do processo de queima a essas temperaturas, transformações químicas ocorrem eliminando os grupos hidroxila $(\mathrm{OH})$ e ativando o carbono presente na SM.

LOBO (2005) relatou em seu trabalho que espectros de materiais carbonosos, apresentam picos característicos na região entre 1000 e $1800 \mathrm{~cm}^{-1}$, materiais grafíticos nessa região referem-se às bandas $\mathrm{D}$ e $\mathrm{G}\left(\sim 1500 \mathrm{a} \sim 1600 \mathrm{~cm}^{-1}\right)$. Para FERRARI et.al (2003), materiais carbonosos apresentam espectros Raman característicos na região entre $800-2000$ $\mathrm{cm}^{-1}$, as chamadas bandas D e G. A banda G refere-se ao alongamento da ligação $\mathrm{sp}^{2}$, já o pico $\mathrm{D}$ está relacionado com a desordem das ligações químicas do material, específicos de carbonos amorfos.

Figura 3 - Comparação dos espectros Raman da CSM em temperaturas de a) $300^{\circ} \mathrm{C}$; b) $400^{\circ} \mathrm{C}$ e c) $500^{\circ} \mathrm{C}$ com tempos de 40,60 e 120 minutos.

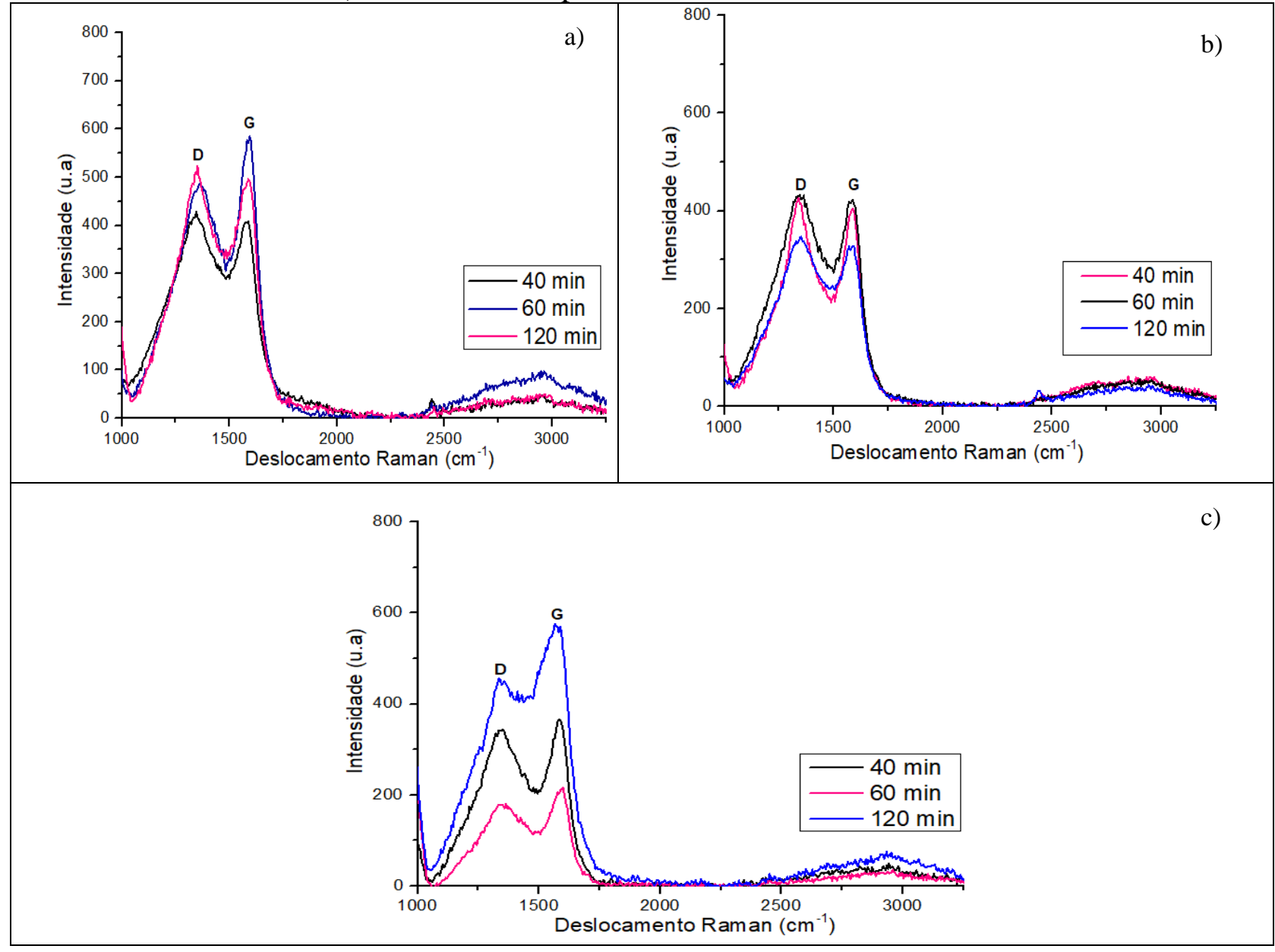




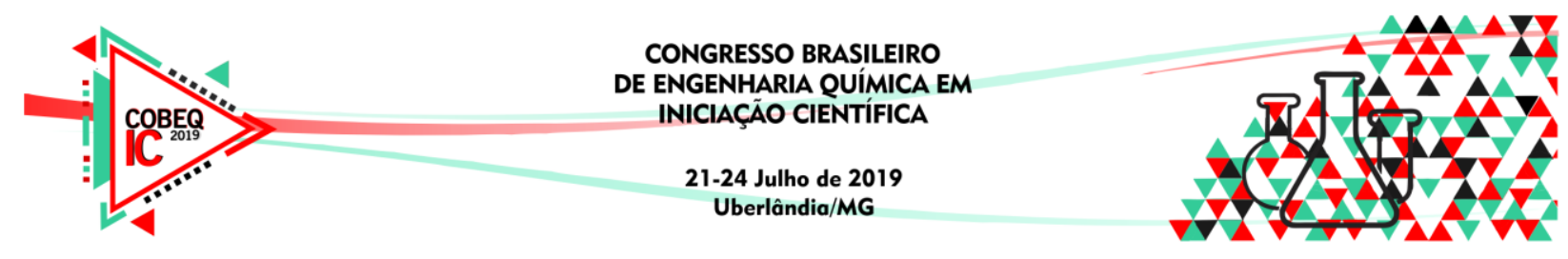

\section{CONCLUSÕES}

Os resultados obtidos com a FRX mostraram a composição química em percentual dos compostos inorgânicos na forma de óxidos presentes nas amostras caracterizadas. Observa-se que as variações de temperatura e tempo dos ensaios realizados, nota-se que a maior variação em porcentagem de massa ocorreu para o óxido de cálcio em uma temperatura de $400^{\circ} \mathrm{C}$ no tempo de 120 min. Porém, a soma da porcentagem dos elementos inorgânicos identificados em cada ensaio representa menos de $20 \%$ da composição percentual em massa da amostra, portanto os $80 \%$ restante da composição do material estão contidos os elementos na qual o equipamento não permite identificar que são: Carbono, Hidrogênio e Oxigênio.

A partir das medidas de espectroscopia Raman da CSM identificou-se a presença da banda $\mathrm{D}\left(\sim 1357 \mathrm{~cm}^{-1}\right)$ e banda $\mathrm{G}\left(\sim 1590 \mathrm{~cm}^{-1}\right)$ nas diferentes temperaturas $\left(300,400\right.$ e $\left.500^{\circ} \mathrm{C}\right)$, sendo estas bandas características de carbono. As maiores intensidades e menores variações observadas para as bandas D e G no espectro Raman, ocorreu em temperatura de $300^{\circ} \mathrm{C}$. Portanto os resultados de FRX e espectroscopia Raman revelam a presença de estruturas de carbono na CSM.

\section{REFERÊNCIAS:}

BERND, M.G.S.; BRAGANÇA, S.R.; HECK. N.; FILHO, L.C.P.S. Synthesis of carbono nanostructures by the pyrolysis of wood sawdust in a tubular. Pos.-Grad. Course of Mines, Metallurgy, and Materials (PPGEM), Fed. Univer. of Rio G. do Sul (UFRGS), Porto Alegre, RS, Brazil, vol.6. Num 2. p.101-012, 2017.

FERRARI, A.C.; RODIL, S.E.; ROBERTSON, J. Interpreration of infrared and Raman spectra of amorphous carbono nitrides. Phys. Rev, v. 67, n.20. 2003.

GEIM, A. K. Graphene: status and prospects. Science, v.324. p.1530 -1543, 2009

LOBO, A, et al. Caracterização De Materiais Carbonosos Por Espectroscopia Raman. Rev. Bras. de Apli. de Vác, v. 24, n. 2, 98-103, 2005.

NOVOSELOV, K.S., FALKO, V.I., COLOMBO, L., GELLERT, P.R., SCHWAB, M.G. and KIM, K. A Roadmap for Graphene. Nature, 490, 192-200, (2012).

PUMERA, M.; AMBROSI, A.; BONANNI, A; CHNG, E. L. K.; POH, H. L. Graphene for eletrochemical sensing and biosensing. Tren. in analy. chemistry. Vol. 29. No. 9, 2010 . 